\title{
Über Metalltrennungen in einem mit Brom beladenen Kohlensäurestrome.
}

\author{
Von \\ P. JANNASCH und ED. Rose. \\ V. Mitteilung. ${ }^{1}$ \\ Mit 3 Figuren im Text.
}

\section{Trennung von Wismut und Kobalt.}

Die im nachstehenden beschriebenen quantitativen Metallbestimmungen bilden die Fortsetzung früherer analoger Trennungen des Wismuts, Zinns und Antimons von Blei und Kadmium. Die inzwischen von uns erreichten Vervollkommnungen der für das Destillationsverfahren erforderlichen Apparate gestatten jetzt ein weit leichteres Arbeiten, als dies in den ersten Anfängen der Brommethode möglich war.

Als Ausgangsmaterial nahmen wir reines metallisches Wismut und Kobaltammonsulfat. Anfänglich hatten wir Versuche mit Kobaltsulfat angestellt, dasselbe erwies sich jedoch wenig brauchbar, da es beim Trocknen an der Luft, sowie beim Aufbewahren zu leicht Wasser verliert und daher meistens zu hohe Resultate liefert.

Den von uns benutzten Apparat zeigt beistehende Figur 1.

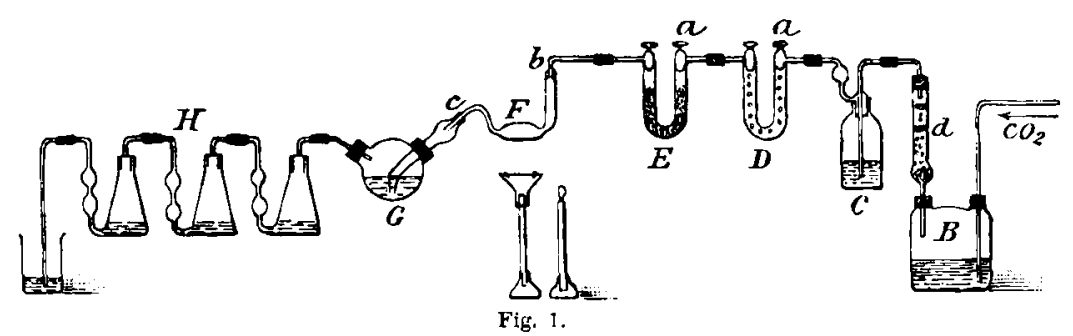

Aus einem KIppschen Apparat geht ein Kohlensäurestrom durch den Trockenapparat $B$ und sättigt sich in der Flasche $C$ mit Brom-

1 Vergl. die früheren Mitteilungen in den Ber. deutsch. chem. Qes. 24, 3746; 25,124 und $736 ; 26,1422$. 
dämpfen, worauf er zum endgültigen Trocknen zwei U-Röhren $D$ und $E$ passiert, von denen die erste mit schwefelsäuregetränkten Glasperlen, die zweite mit Glaswolle zum Auffangen von mechanisch fortgerissenen Schwefelsäurespuren gefüllt ist. Beide Röhren sind mit Glashähnen versehen. Nun gelangt der Strom durch ein bei $b$ eingeschliffenes Einleitungsrohr in das Destilliergefäfs $F$, welches die sulfurierte Substanz (s. unten) enthält. Das ron $F$ nach der Vorlage $G$ gehende Verbindungsrohr ist bei $c$ aufgeschliffen und besitzt eine kugelförmige Erweiterung, durch welche das Entwickelungsrohr, wie es Figur 2 zeigt, hindurchgeht. Diese Vorrichtung hat den Zweck, das Hineingelangen von Wismutbromid in die Schliffstelle zu vermeiden, aus welcher es sich nur schwer wieder völlig austreiben lälst. Die Vorlage $G$ ist mit $150 \mathrm{ccm}$ verdünnter Salpetersäure $(1: 10)$ gefüllt. Auf diese folgen drei Volmardsche Vorlagen mit eingeschliffenen Glasrohrstopfen, von denen die beiden ersten verdünnte Salpetersäure enthalten, während die letzte zur Absorption des überschüssigen Broms nur mit Alkohol beschickt ist. Ein Ableitungsrohr taucht in ein Becherglas mit etwas Alkohol.

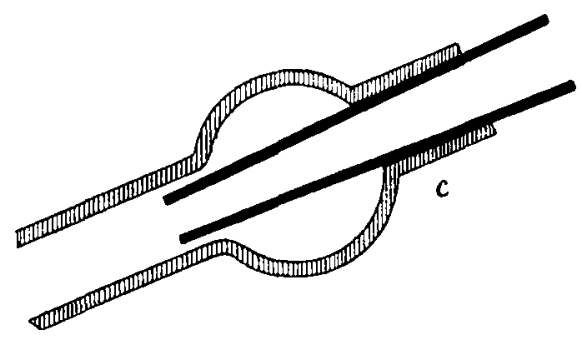

Fig. 2.

Die möglichst fein gepulverte Substanz wird entweder im Wägegläschen tariert (je 0.3-0.5 g), oder im Apparate selbst abgewogen. $\mathrm{Zu}$ letzterem $\mathrm{Zwecke}$ versieht man denselben mit einer Aufhängevorrichtung aus Platindraht. Man leitet nun über die Substanz einen trockenen Kohlensäurestrom ${ }^{1}$ und erwärmt zuerst in Luftbade, dann, wenn nötig, direkt durch Fächeln mit einem Flachbrenner. Hierdurch wird alles Krystallwasser ausgetrieben und in dem gegebenen Falle auch der gröfste Teil des vorhandenen Ammonsalzes. ${ }^{2}$ Ist

1 Derselbe muईs sicher salzsäurefrei sein, weshalb man zweckmälsig den oberen Teil des Chlorcalciumrohres $d$ mit Doppelspatstiuckchen ausfüllt.

${ }^{2}$ Dasselbe wirkt aber keineswegs störend auf den Trennungsgang eir. 
dieses geschehen, so wird die Substanz mit 3-5 g reinem Schwefelpulver ${ }^{1}$ überschüttet und durch Schütteln möglichst innig damit gemischt, worauf die Erhitzung der Masse in einem trockenen Schwefelwasserstoffstrome erfolgt. Nach ruhigem Flusse des Gemisches sublimiert man den überschüssigen Schwefel ab und lälst im Schwefelwasserstoffstrome erkalten. Beim Schütteln und Klopfen fällt der Rückstand zu einem Pulver zusammen, welches man abermals mit überschïssigem Schwefel zur Wiederholung der ganzen Operation mengt. Nach dem Erkalten schaltet man das Rohr mit den Sulfiden in den Bromierungsapparat ein. Die Schliffstelle $c$ kann eventuell mit einer Spur Vaselin bestrichen werden, meist sind indessen die Apparate so gut eingeschliffen, dal's man die Anwendung eines Einfettungsmittels besser vermeidet. Nun lälst man einen nicht zu langsamen Bromkohlensäurestrom über die Substanz gehen. ${ }^{2}$ Ist alle Luft verdrängt, so erhitzt man durch Fächeln mit dem Flachbrenner. Es tritt sehr bald ein ruhiges Absublimieren zuerst von Bromschwefel, dann von Wismutbromid ein. Man thut gut, unter das rechtwinklige Eintrittsknierohr in entsprechender Entfernung ein kleines Flämmchen zu stellen, um dem Zurücksteigen von Wismutbromiddämpfen vorzubeugen. Geht die Hitze mit der Spaltflamme cinmal zu hoch, so treten im Apparate schillernde Flitter ron Kobaltbromür auf. Alsdann mufs man sofort den Strom abstellen und einige Augenblicke erkalten lassen. In dem Ableitungsrohre setzt sich das Wismutbromid als gelbbrauner Beschlag an. Diesen vertreibt man von Zeit zu Zeit, um beurteilen zu können, ob die Abdestillation beendet ist. Zeigt sich kein neuer Beschlag mehr bei weiterem Erhitzen, so schliefst man den Kippschen Apparat ab, schaltet die Bromflasche aus, stellt die Verbindung von neuem her und läfst im Kohlensäurestrome erkalten. Hierauf nimmt man das Destillierrohr heraus, mengt den Inhalt desselben durch Schütteln gut durch und erhitzt ihn noch einmal kurze Zeit im Bromstrome, um sicher zu sein, dafs auch alles Wismutbromid vollständig übergegangen war. Bei dem Fächeln mit dem Flachbrenner mufs man rorsichtig zu Werke gehen, danit sich der Schliffstellenverschluls nicht durch Anstolsen des Brenners an das Rohr lockert. Das entstandene grüne Kobaltbromid ist bis auf einen schwarzen Rückstand

1 Die Anwendung von jodhaltigem Schwefel war hier nicht erforderlich (vergl. weiter unten bei 3. .)

${ }^{2}$ Derselbe muls absolut frei scin von Wasser, Chlor und Salzsäure. 
in Wasser leicht löslich. Letzterer wird von verdünnter Salzsäure schwer angegriffen, allein er löst sich bei Zusatz von rauchender Salpetersäure oder Königswasser. Das zurückbleibende Kobalt enthält kein Wismut, was man beweisen kann, wenn man die Lösung auf dem Wasserbade eben zur Trockne rerdampft und mit Wasser aufnimmt, wobei keinerlei Trübungen zu beobachten sind. Die stark verdünnte Lösung wird in einer Porzellanschale zum Sieden erhitzt und mit einem möglichst geringen Überschusse von Natriumhydroxyd versetzt. Das abfiltrierte und mit heifsem Wasser ausgewaschene Kobalthydroxyd trockneten wir bei $75-80^{\circ}$, rieben es von dom Filter möglichst sorgfältig $a b$ und reraschten das Filter für sich. Dann fügten wir den Kobaltniederschlag hinzu, glühten zur Konstanz und wogen ihn als $\mathrm{Co}_{3} \mathrm{O}_{4}$ und als Co.

Das Wismut aus den drei Salpetersäurevorlagen wird in eine Porzellanschale gebracht und auf dem Wasserbade behufs Wegtreibens des Broms etc. zur Trockne verdampft. Man löst hierauf mit verdünnter Salpetersäure und filtriert von etwas ausgeschiedenem Schwefel ab, dafür Sorge tragend, das Filter mit der warmen Säure so lange nachzuwaschen, bis ein auf dem Platinbleche verdampfter Tropfen keinen Rückstand mehr hinterläist. Hierauf fällt man das Wismut mit einem Überschusse von Ammoniak und Wasserstoffsuperoxyd und behandelt den ausfallenden Niederschlag, wie wir es schon früher ${ }^{1}$ ausführlich beschrieben haben. Ist zum Lösen und Auswaschen des Filters sehr viel Säure verwendet worden, so thut man gut, die Lösung vor dem Fällen zur Verjagung des Säureüberschusses nochmals einzudampfen. Das im Platintiegel gewogene Wismutoxyd prüfe man stets auf ein etwaiges Vorhandensein von Kieselsäure. $^{2}$

In dem Filtrate des Wismutniederschlages erhielten wir mit Schwefelammonium nicht eine Spur von Braunfärbung. Mithin war das von uns erhaltene Wismut frei von Kobalt. Das mit starker Salzsäure im Tiegel überschüttete Kobaltoxydoxydul wurde auf dem

1 Ber. deutsch. chem. Ges. 27, 14.

${ }^{2}$ Früher wurde das $W$ ismut in dem erhaltenen Destillat durch Ver. dampfung desselben und Wägung des Rückstandes im Porzellantiegel bestimmt (vergl. Ber. deutsch. chem. Ges. 25, 124). Nach Auffindung der ausgezeichneten Fällungsmethode des Wismuts mit Wasserstoffsuperoxyd verdient die obige Behandlungsweise der Vorlageflüssigkeiten offenbar den Vorzug. Wir hoffen jedoch auch das ältere Verfahren durch endgültiges Ausglühen des blofsen Verdampfungsrückstandes in einem I'latintiegel entsprechend v'rbessern zu können. 
Wasserbade eingetrocknet, sodann unter Zusatz von 2-3 Tropfen verdünnter Salpetersäure vollkommen klar gelöst und diese Flüssigkeit mit Wasser stark verdünnt. Es entstand hierbei nicht die mindeste Trübung, ebensowenig ein Niederschlag mit überschüssigem Ammoniak, ein Beweis ron dem Freisein unseres Präparates an Wismutrerunreinigungen. ${ }^{1}$

Analyse I. $-0.4518 \mathrm{~g} \mathrm{Bi}+0.4248 \mathrm{~g} \mathrm{Co}\left(\mathrm{NH}_{4}\right)_{2}\left(\mathrm{SO}_{4}\right)_{2}+6 \mathrm{H}_{2} \mathrm{O}=0.8766$ angewandte Substanz gaben $=0.5042 \mathrm{~g} \mathrm{Bi}_{2} \mathrm{O}_{3}=0.4522 \mathrm{~g} \mathrm{Bi}$ und $0.0864 \mathrm{~g} \mathrm{Co}_{3} \mathrm{O}_{4}$ (Theorie $=0.0861 \mathrm{~g}$ ). Auf die Gesamtmenge bezogen erhält man $=51.59 \% \mathrm{Bi}$ und $9.86 \% \mathrm{Co}_{3} \mathrm{O}_{4}$ (Theorie $=51.54 \% \mathrm{Bi}$ und $9.82 \% \mathrm{Co}_{8} \mathrm{O}_{4}$ ).

Analyse II. $\left.-0.3578 \mathrm{~g} \mathrm{Bi}+0.4119 \mathrm{~g} \mathrm{Co}\left(\mathrm{NH}_{4}\right)_{2} \mathrm{SO}_{4}\right)_{2}+6 \mathrm{H}_{2} \mathrm{O}=0.7697$ angewandte Substanz gaben $=0.4007 \mathrm{~g} \mathrm{Bi}_{2} \mathrm{O}_{3}=0.3594 \mathrm{~g} \mathrm{Bi}$ und $0.0620 \mathrm{~g} \mathrm{Co}_{0}$ (Theorie $=0.0613 \mathrm{~g}$ ). Auf die Gesamtmenge bezogen erhălt man $=46.69 \% \mathrm{Bi}$ und $8.06 \%$ Co (Theorie $=46.49 \% \mathrm{Bi}$ und $7.96 \% \mathrm{Co}$ ).

\section{Trennung von Wismut und Nickel.}

Diese Trennung erfolgt genau in derselben Weise wie diejenige von Wismut und Kobalt. Die nach der Brombehandlung im Glasrohr zurückbleibende krystallinische Nickelverbindung ist in Wasser schwer löslich, dagegen sehr leicht bei einem Zusatz von Säure. Auch hier war die Trennung eine vollkommene.

Analyse I. $-0.4565 \mathrm{~g} \mathrm{Bi}+0.4670 \mathrm{~g} \mathrm{Ni}\left(\mathrm{NH}_{4}\right)_{2}\left(\mathrm{SO}_{4}\right)_{2}+6 \mathrm{H}_{2} \mathrm{O}=0.9235 \mathrm{~g}$

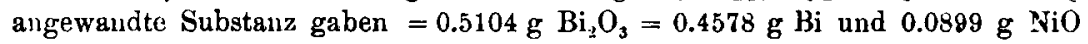
(Theorie $=0.0883 \mathrm{~g}$ ). Auf die Gesamtmenge bezogen erhält man $49.57 \% \mathrm{Bi}$ und $9.73^{\circ} \%_{0} \mathrm{NiO}$ (Theorie $=49.43 \%$ Bi und $9.56 \% \mathrm{NiO}$ ).

Analyse II. $-0.5384 \mathrm{~g} \mathrm{Bi}+0.5102 \mathrm{~g} \mathrm{Ni}\left(\mathrm{NH}_{4}\right)_{2}\left(\mathrm{SO}_{4}\right)_{2}+6 \mathrm{H}_{2} \mathrm{O}=1.0486 \mathrm{~g}$ angewandte Substanz gaben $=0.5991 \mathrm{~g} \mathrm{Bi}_{2} \mathrm{O}_{3}=0.5374 \mathrm{~g} \mathrm{Bi}$ und $0.0959 \mathrm{~g} \mathrm{NiO}$ (Theorie $=0.0965 \mathrm{~g}$ ). Auf die Gesamtmenge bezogen erhält man $51.25 \% \mathrm{Bi}$ und $9.15 \% \mathrm{NiO}$ (Theorie $=51.34 \% \mathrm{Bi}$ und $9.20 \% \mathrm{NiO}$ ).

1 Wir möchten hier nicht unerwähnt lassen, dafs die Wägung des Kobalts in der Form von Metall durch Wasserstoffreduktion nur dann brauchbare Werte giebt, weun das im Kippschen Apparate entwickelte Gas absolut rein ist. Wir benutzen zur Erreichung dieses Zweckes nur ehemisch reines Zink und platinchloridhaltige Schwefelsäure $(1: 4)$ und waschen das Gas vor seinem eigentlichen Eintritte in einen sauberen Trockenapparat (am Ausgange mit festem Kalihydrat) durch Lösungen von Blei (alkalisch), Silbernitrat und Kaliumpermanganat. Aber auch diese Vorsichtsmalsregeln erweisen sich bei raschen Strömen als nicht ausreichend genug, weshalb wir am Schlufs noch eine ca. $15 \mathrm{~cm}$ lange Kupferdrahtnetzspirale vorlegen, welche sich in einem auf beiden Seiten ausgezogenen Kaliglasrohr befindet und in einem Gasbrennerofen zur Hälfte mäfsig stark und zunächst dem Roseschen Tiegel nur ganz gelinde exhitzt wird. Endlich ist es praktisch, zum Einleiten des Wasserstoffes ein im kurzen Sclienkel mit Kugelerweiterung versehenes Kaliglasrohr anzuwenden, das bis in die Tiegelmitte hineinreicht. 


\section{Die Trennung des Zinns und Antimons von Blei und Kupfer.}

Von P. Jannasch und R. Niederhof heim.

Diese quantitativen Trennungen auf dem Wege der trockener Destillation sind von uns der Abwechselung und des Vergleiches wegen in einem längeren Einschliffrohr aus Kaliglas ausgeführt worden in Verbindung mit der durch Fjg. 3 illustrierten Absorptionsvorrichtung. Das betreffende Rohr besitzt eine Gesamtlänge von $34.5 \mathrm{~cm}$ bei einer Innenweite von $15 \mathrm{~mm}$ und einer Glasdicke von $1 \mathrm{~mm}$. Der stumpfwinklig abgebogene Teil desselben beträgt $19.5-20 \mathrm{~cm}$, wovon auf das feste Obenende $5 \mathrm{~cm}$ bei $4^{3} / 4-5 \mathrm{~mm}$ Lichtenweite am Endpunkte kommen, während das ca. $15 \mathrm{~cm}$ lange Aufschliffrohr oben 10 and unten $8 \mathrm{~mm}$ Weite hat. Die Schliffstelle $^{1}$ selbst mifst $23 \mathrm{~mm}$ und ragt $2.5 \mathrm{~cm}$ aus dem Verbindungs.

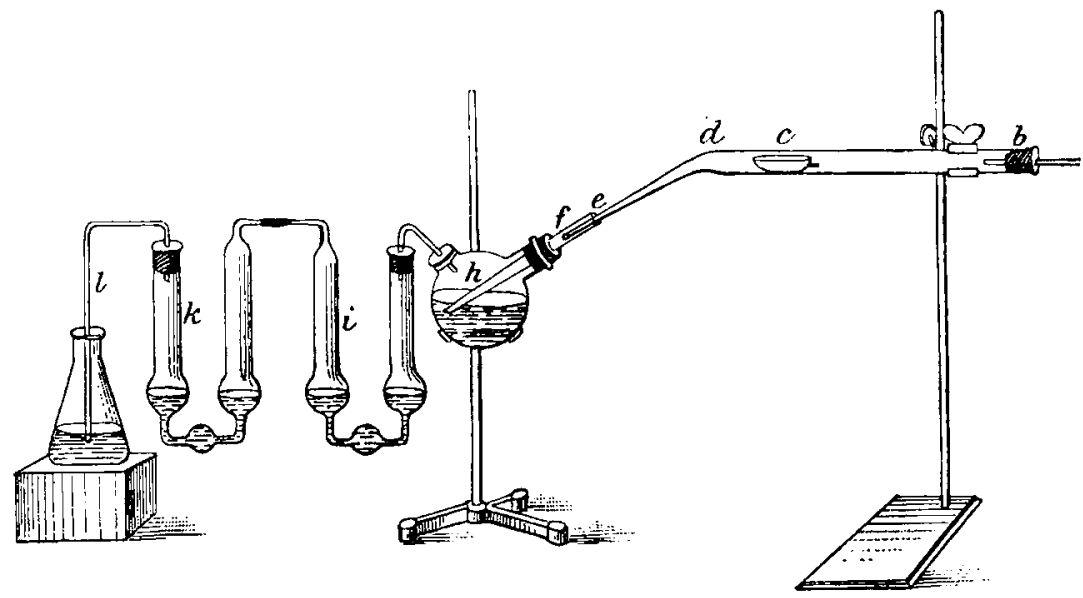

Fig. 3.

kork hervor. Das die angewandte Substanz enthaltende, $7.2 \mathrm{~cm}$ lange (ohne Griff) und mindestens $5 \mathrm{ccm}$ Raum fassende Porzellanschiffchen $c$ steht von der Bugstelle $d 7.5 \mathrm{~cm}$ entfernt, so dals für den leeren Teil der Röhre 18-19 cm verbleiben. Die Stelle der VolHaRdschen Absorptionskölbchen wird durch zwei Ptusigot-Röhren ersetzt, während den Schlufs des Ganzen ein mit Natronlauge beschickter Erlenmeyer bildet. Die Vorlagen $h, i$ und $k$ enthalten

1 Wir empfehlen auch hier die Anbringung einer Kugelerweiterung wie bei 1 als eine ganz wesentliche Verbesserung für die Abdestillation der flüchtigen Bromide. 
verdünnte Salpetersäure $(1: 5)$. Obige Absorptionsvorrichtung ist leicht zu beschaffen und vollkommen ausreichend, die bei den Metalltrennungen in 1 und 2 empfohlene Art aber angenehmer im Gebrauch und in jeder Hinsicht fehlerfrei. Die der Bromierung vorausgehende Sulfurierung des vorliegenden Gemenges erfolgte hier stets unter Benutzung von jodhaltigem Schwefel. ${ }^{1}$ Das zur Bromierung dienende Brom muls chlor- und wasserfrei, der Kohlensäurestrom salzsäurefrei sein. ${ }^{2}$

\section{a. Trennung von Zinn und Blei. ${ }^{3}$}

Angewandt wurden zu dieser Trennung Bleinitrat und Zinnsäureanhydrid (vorher ausgeglüht). Wir fanden hierbei alle schon früber gemachten Angaben vollkommen bestätigt. Das Bleinitrat spritzt sehr leicht bei der Sulfurierung, was sich aber vermeiden lälst, wenn man es vor der Abwägung sehr fein pulvert. Die Sulfurierung wurde der Sicherheit wegen stets zweimal vorgenommen. Die Bromierung erfolgt nun, wie wir es näher in 1 beschrieben haben. Das im Schiffchen zurückbleibende geschmolzene Bleibromid kann bereits als solches gewogen werden. Dasselbe muls sich vollständig klar in. frischem Chlorwasser auflösen. Zur Kontrolle fällt man das Blei am besten aus dieser Lösung mit ammoniakalischem Wasserstoffsuperoxyd, um es schliefslich als Bleioxyd im Platintiegel zu bestimmen. ${ }^{1}$ Das so erhaltene Oxyd darf bei seiner Lösung in verdünnter Salpetersäure keinen Rückstand von Zinnsäure hinterlassen.

\section{b. Trennung von Zinn und Kupfer.}

Je $0.35-0.5 \mathrm{~g}$ zerriebener Kupfervitriol und Zinnoxyd wurden zunächst nach der Abwägung im Porzellanschiffchen gemengt, eine Stunde auf $150-170^{\circ}$ erhitzt, darnach zweimal sulfuriert und schliefslich im Bromstrome geglüht. Zuerst geht wesentlich Bromschwefel über, dem rasch das sehr flüchtige Zinubromid folgt. Nach etwa einer halben Stunde fängt die Substanz im Schiffchen zu schmelzen an und die Dampfbildung hört nach und nach auf, wodurch sich das Ende der Reaktion anzeigt. Es ist ratsam, jetzt den Bromentwickler auszuschalten und einige Zeit nur trocken Kohlensäure

1 Ber. deutsch. chem. Gies. 26, 1422.

${ }^{2}$ Vergl. die eingangs citierten Abhandlungen.

${ }^{3}$ Ber. deutsch. chem. Ges, 26, 1424.

- Diese Zeitschr. 8, 302. Die Bestimmung als Bloisulfat dauert jedenfalls länger. 
durch den Apparat zu leiten, um danach die Vorlagenflüssigkeit ohne zu starke Belästigung durch Bromdämpfe sammeln zu können. Die in einer grofsen Porzellanschale vereinigten Lösungen von $h$, $i$ und $k$ werden zunächst bis auf ein kleines Volumen eingedampft, dann zusammen mit der ausgeschiedenen Zinnsäure in einen grölseren gewogenen Berliner Porzellantiegel von $40-45 \mathrm{ccm}$ Inhalt gebracht, auf dem Dampfbade von Wasser und im offenen Luftbade von Schwefelsäure befreit, worauf man den Rückstand von Zinnsäure bis zur Gewichtskonstanz glüht, nötigenfalls unter einer vorausgehenden Durchfeuchtung mit konz. Salpetersäure. Hält man unter gegebenen Verhältnissen die Fällungsmethode zur Bestimmung des Zinns für unfehlbarer, so übersättigt man die erhaltene Lösung (unmittelbar nach deren Aufsammlung) mit Ammoniak, fügt überschïssiges Ammonsulfid hinzu und säuert wieder mit verdünnter Salzsäure an. Das ausgeschiedene Zinnsulfid wird filtriert, mit schwefelwasserstoffhaltigem fünfprozentigen Ammonnitrat gewaschen, getrocknet, geglüht und als Zinndioxyd gewogen.

Das im Schiffchen befindliche Kupferbromid löst man in verdünnter absolut reiner Salpetersäure und dampft diese Flüssigkeit in einem geräumigen Porzellantiegel so lange unter erneuter Aufnahme des restierenden Salzes mit der konz. Säure ein, bis alles Bromid vollständig in Nitrat verwandelt ist, worauf man glüht (anfänglich im offenen Luftbade) und wägt; oder man fällt die ursprüngliche salpetersaure Lösung direkt mit Natron in der Kochhitze etc. Eine direkte Wägung des Kupfers im Schiffchen ist unmöglich, da es nicht in der Form von reinem Bromid zurückbleibt, sondern vermischt mit gewissen Mengen von Bromür.

Analyse. $-0.4132 \mathrm{~g} \mathrm{SnO}_{2}+0.4132 \mathrm{~g} \mathrm{CuSO}_{4} .5 \mathrm{H}_{2} \mathrm{O}=0.8264 \mathrm{~g}$ angewandte Substanz lieferten $=0.4140 \mathrm{~g} \mathrm{SnO}_{2}$ und $0.1302 \mathrm{~g} \mathrm{CuO}$ (Theorie $=0.1314 \mathrm{~g}$ ). Auf die gesamte angewandte Substanz bezogen ergiebt sich hieraus:

$\begin{array}{cc}\text { Gefunden: } & \text { Berechnet: } \\ \mathrm{SnO}_{\mathbf{2}}=50.10 \% & \mathbf{5 0 . 0 0 \%} \\ \mathrm{CuO}=\mathbf{1 5 . 7 6} \% & \mathbf{1 5 . 9 0 \%}\end{array}$

c. Trennung von Antimon und Blei.

Bei der Sulfurierung des hier zur Verwendung gelangten Gemisches von Antimonpulver und Bleinitrat mufs man entsprechend vorsichtiger verfahren als bei der vorherigen Trennung, weil sich sonst minimale Mengen von Schwefelantimon verflüchtigen können, erkenutlich an kleinen rötlichen Flecken im Rohr. Am besten stellt man eine möglichst kleine Flamme unter das in der Mitte der Röhre 
befindliche Schiffchen und verjagt den überschüssigen Schwefel ganz lingsam. Obwohl sich das metallische Antimon leichter als geglühtes Zinndioxyd sulfuriert, ist trotzdem anzuraten, die Sulfurierung der angewandten Substanz auch hier zu wiederholen und überhaupt, der eben erwähnten geringen Flüchtigkeit des Schwefelantimons lalber, in demselben Rohr vorzunehmen, worin man später bromiert. Das gebildete Antimonbromid geht sehr leicht als gelber Dampf über und löst sich in der Vorlage völlig auf. Man erhitzt so lange, bis keine Dämpfe mehr entweichen. Sollten sich geringe Mengen von nichtflüchtiger Antimonverbindung im Rohr selbst irgendwo festsetzen, so entfernt man dieselben am besten mit bromhaltiger Salpetersäure. Die Vorlagefliussigkeiten werden genau so behandelt, wie wir es in erster Linie bei dem Zinn näher angegeben haben. Nach dem Abrauchen aller überschiüssigen Schwefelsäure muls man den Rückstand zwei- bis dreimal mit starker rauchender Salpetersäure von 1.5 D. eintrocknen, ehe man glüht und als Antimonylmetaantimoniat wägt. Hat man das Antimon aus den vereinigten Vorlageflüssigkeiten als Antimonsulfid abgeschieden (vgl. bei b), so führt man alsdann diese auf einem Filter gesammelte und mit einer $5 \%$ igen schwefelwasserstoffhaltigen Ammonnitratlösung ausgewaschene Füllung auf die folgende Weise in $\mathrm{Sb}_{2} \mathrm{O}_{4}$ über. Man trocknet zunächst den Niederschlag gut bei mälsiger Hitze $\left(80-90^{\circ}\right)$, giebt ihn recht vollständig von dem Filter losgelöst in einen grofsen Porzellantiegel und extrahiert schliefslich das auf einer Uhrschale ausgebreitete Filter mit einer kleinen, im Reagensrohr zum Kochen erhitzten Menge Ammonsulfid ${ }^{1}$ unter Nachwaschung mit der Spritzflasche. Nun wird die Flüssigkeit im Tiegel, welcher bei geschickter Ausführung der Manipulation etwa bis zu zwei Drittel davon angefüllt ist, auf dem Wasserbade zur Trockne eingedampft, worauf man denselben in ein Glasdreieck über darunter befindliche rauchende Salpetersäure von $1.5 \mathrm{D}$. stellt und das Ganze mit einem Becherglase bedeckt $6-12$ Stunden ruhig stehen lälst. Die zur allmählichen Oxydation des Schwefelantimons bestimmte Salpetersäure befindet sich zweckmälsig in einer Krystallisierschale und letztere selbst auf einem flachen Porzellanteller. Am Ende überschüttet man das entstandene völlig weifse Dioxyd direkt mit der starken Säure, verdampft auf dem Wasserbade, jagt die überschüssige Schwefelsäure im Luftbade fort, glüht und wägt bis zur Gewichtskonstanz, event. unter erneutem Zusatz von etwas Säure u. s. f.

\footnotetext{
' Frisch aus reinem Ammoniak za bereiten.
} 
Eine Überführung des mit Salzsäure gefällten und nur mit warmem Schwefelwasserstoffwasser gewaschenen Gemisches ron $\mathrm{Sb}_{2} \mathrm{~S}_{3}+\mathrm{Sb}_{2} \mathrm{~S}_{5}+\mathrm{S}$ in reines $\mathrm{Sb}_{2} \mathrm{~S}_{3}$ kann gleichfalls in einem Porzellantiegel geschehen, indem man es wie oben in denselben bringt, alsdann das Wasser verdampft, mit einem durchbohrten Deckel verschliefst und im Kohlensäurestrome bei Luftbadtemperatur ${ }^{1}$ erhitzt, bis kein Schwefel etc. mehr entweicht.

Das im Schiffchen zurückgebliebene Bleibromid wird wie früher beschrieben weiter verarbeitet.

Analyse. $-0.4294 \mathrm{~g} \mathrm{Sb}+0.3642 \mathrm{~g} \mathrm{~Pb}\left(\mathrm{NO}_{3}\right)_{2}=0.7936 \mathrm{~g}$ angewandte Substanz gaben $=0.5419 \mathrm{~g} \mathrm{Sb}_{z} \mathrm{O}_{4}=0.4283 \mathrm{~g} \mathrm{Sb}$ und $0.2446 \mathrm{~g}$ PbO (Theorie $=0.2453$ ).

$\begin{array}{cc}\text { Gefunden: } & \text { Berechnet: } \\ \mathrm{Sb}=54.11 \% & 53.97 \% \\ \mathrm{PbO}=30.82 \% & 30.91 \%\end{array}$

d. Trennung von Antimon und Kupfer.

Zur Verwendung gelangte ein Gemisch von metallischem Antimon und ron Kupfervitriol. Nach der Vertreibung des Wassers und der Sulfurierung leitet man so lange Brom über die erhitzte Substanz, bis das zurückbleibende Bromkupfer geschmolzen ist und sich keine Antimonbromiddämpfe mehr entwickeln. Man vermeide bei dem Destillationsprozesse jede unnötige Überhitzung, da das Kupferbromid etwas flüchtiger ist als das Bleibromid.

Analyse. $-0.3864 \mathrm{~g} \mathrm{Sb}+0.5186 \mathrm{~g} \mathrm{CuSO}_{4} .5 \mathrm{H}_{2} \mathrm{O}=0.9050 \mathrm{~g}$ angewandte $\mathrm{Sub}-$ stanz gaben $0.4882 \mathrm{~g} \mathrm{Sb}_{2} \mathrm{O}_{4}=0.3858 \mathrm{~g} \mathrm{Sb}$ und $0.1634 \mathrm{~g} \mathrm{CuO}$ (Theorie $=0.1650$ )

$$
\begin{array}{rc}
\text { Gefunden: } & \text { Berechnet: } \\
\mathrm{Sb}=42.63 \% & 42.69 \% \\
\mathrm{CuO}=18.05 \% & 18.23 \%
\end{array}
$$

Die von uns im Obigen aufgeführten Analysenbeispiele dürften die Ausführbarkeit und Richtigkeit der Sulfidbromierungsmethode zur Genüge darthun. Sie hat insofern noch den Charakter des Neuen, als es sich hierbei um quantitative Trennungen handelt, bei welchen weder ein Lösen des angewandten Gemisches, noch eine darauffolgende Ausfällung stattfindet; es sind also in ihrem Grundprinzip Analysen ohne Auflösung, Fällung und Filtration, welche in letzter Linie nur Verdampfungen und Glühoperationen erfordern. Freilich ist dieses Ideal nicht immer praktisch erreich-

1 Wir bedienen uns hierzu entspreehend grofser Nickclbecher und eines Fletcher-Flachbrenners (vgl. Ber. deutsch. chem. Ges. 26, 1497). Am geeignetsten erwiesen sich hohe Meifsener Tiegel von 45 oder 65 cem Inhalt, deren Deckel wir mit einer Durehbohrung versehen lielsen. 
bar, was einerseits in den die Analyse begleitenden Nebenumständen wie $\mathrm{A}$ bfiltrieren von Beimengungen $\left(\mathrm{SiO}_{2}\right.$ etc.) begründet liegt, andererseits bis auf weiteres in der Bevorzugung der genauesten und sichersten Bestimmungsform des flüchtigen oder des nichtflüchtigen Metalles, wie derjenigen des Wismuts durch Ausfällung als Hyperoxydhydrat, gegenüber dem blofsen Abdampfungs- und Ausglühverfahren. Wir haben auch noch weitere Versuche unternommen zur Aufklärung der mit der Brommethode im Zusammenhange stehenden Verbältnisse, z. B. der Möglichkeit einer direkten Verflüchtigung von fein gepulvertem Antimon, Arsen etc. in einem Bromstrome, allein mit negativem Ergebnis. Bei dem Arsen ist die trockene Sulfurierung ausgeschlossen, da sich dessen Sulfide bei der Temperatur des siedenden Schwefels verflüchtigen. Indes bietet diese Eigenschaft wiederum ein Mittel dar, um das Arsen von Blei, Kupfer u. s. f. indirekt zu trennen. Die vorliegende Substanz wird alsdann mehrmals mit reinem Schwefel im trockenen Schwefelwasserstoffstrome erhitzt, das entstehende Arsensulfid zugleich mit dem überschüssigen Schwefel vertrieben und das restierende nicht flüchtige Metallsultid für sich weiter verarbeitet. Durch eine besondere Behandlung der flüchtigen Anteile mit Schwefelkohlenstoff, Ammoniak etc. läfst sich auch das Arsen zum Zwecke seiner Wägung daraus quantitativ isolieren.

Was nun den zu benutzenden Apparat anlangt, so geben wir im allgemeinen dem in 1 beschriebenen Doppelknierohr gegenüber der Langröhre den Vorzug. Die letztere wird sich indessen vorteilhaft bei Antimontrennungen verwerten lassen. Aufserdem ist es rationeller, die Bromierung mit dem Spaltbrenner vorzunehmen, während dieses bei der Sulfurierung weniger notwendig erscheint.

Gegenwärtig sind wir damit beschäftigt, den Sulfurierungs- und den Bromierungsprozels dadurch zu rereinigen, dafs wir über die gegebene Substanz unter Erwärmen einen schwefelbromidhaltigen Kohlensäurestrom leiten. Aufserdem haben wir Destillationsversuche bei bestimmten Temperaturen angestellt, wozu wir uns eines besonderen, aus Nickelmetall hergestellten geschlossenen Luftbades bedienen.

Heidelberg, Univ.-Laboratorium, April 1895.

Bei der Redaktion eingegangen am 6. April 1895. 